\title{
Exploring Simulation-Based Configuration Decisions
}

\author{
Michael Smit and Eleni Stroulia* \\ Department of Computing Science \\ University of Alberta \\ Edmonton, AB, Canada \\ \{msmit, stroulia\}@cs.ualberta.ca
}

\begin{abstract}
As service compositions grow larger and more complex, so does the challenge of configuring the underlying hardware infrastructure on which the component services are deployed. With more configuration options (virtualized systems, cloud-based systems, etc.), the challenge grows more difficult. Configuring service-oriented systems involves balancing a competing set of priorities and choosing trade-offs to achieve high-priority goals. We describe a simulation-based methodology for supporting administrators in making these decisions by providing them with relevant information obtained using inexpensive simulationgenerated data. From our existing services-aware simulation framework, we generated millions of performance metrics for a given system in varying configurations. We describe how we structured our simulation experiments to answer specific questions such as optimal service distribution across multiple servers; we relate a general methodology for assisting administrators in balancing trade-offs; and we present results establishing benchmarks for the cost and performance improvements we can expect from run-time configuration adaptation for this application.
\end{abstract}

Deciding on the size, scale, and configuration of computing infrastructure is complex even for technical experts. Decisions must be made about trade-offs between many different attributes and configuration parameters. Configuration assistance supports decision-making about systems; our focus is on the domain of service-oriented software infrastructure, particularly trade-offs involving quality versus cost, where "quality" means attributes that fall within the domain of quality of service (QoS), and "cost" means financial cost.

We offer three novel contributions in the area of configuration assistance, each on a path toward autonomic assistance based on models built by reasoning with simulation-generated data. These methodologies are applicable to other applications and data sets. We describe a proof-of-concept based on data generated by a simulated service-oriented system (running on our existing simulation framework [12]), run in a variety of configurations and usage scenarios.

The first contribution is question answering, where the simulation is run in specific scenarios designed to explore the system behavior under alternative conditions. For example, we considered the challenge of distributing the operations

\footnotetext{
^ The authors acknowledge the generous support of iCORE, NSERC, and IBM.
} 
of a web service among a number of servers to identify the configuration that produced the best performance versus cost trade-off. For all possible distributions on 1,2 , or 3 servers, we simulated a two hour block of realistic traffic. The single-server configuration took 277 minutes to process all of the requests. The fastest two-server configurations took 140 minutes (50\% less time); twice the cost, twice the performance. The fastest three-server configurations completed shortly after the traffic generator stopped generating requests (120 minutes), $57 \%$ less time than the single server. This represents 3 times the cost but only 2.3 times the performance: a more expensive improvement. An expert manually created a three-server configuration using the same information available to the simulation. His suggestion took 137 minutes: worse than the best three-server configurations but better than two servers.

Second, we present our method for undirected exploration of potentially conflicting configuration goals and trade-offs, where we assist in establishing service-level agreements (SLAs), appropriate thresholds, accurate costing models, or appropriate configurations to meet a specific SLA or cost model. The foundation is our visual comparison tool for viewing the trade-off between two potentially conflicting metrics. For example, to identify an SLA with appropriate thresholds, a user is shown a series of such comparisons for relevant metrics, and for each selects the configurations with the balance they desire. Each configuration is given a fitness score based not only on how frequently that configuration is selected, but also by both how close to the "ideal" trade-offs identified by the user and how important each trade-off is to the user (estimated automatically). This identified plausible ideal configurations; a full user study of satisfaction with the results is pending.

Finally, we explored the effect of run-time configuration adaptation on the simulated application, determining the benefit of and targets for a future autonomic system. The tool developed for this exploration could also be used to train administrators to adapt configurations to changing situations, or to help administrators understand how the system behaves in various configurations. Using our simulation, we sent an identical set of requests to 5 configurations: fixed $3,4,5$, and 6 server configurations, and a configuration where the number of servers was manually adapted based on the performance of the application. Compared to a fixed 3-server configuration, a manually varied configuration with 1 to 6 servers was $3 \%$ more expensive but performed $75 \%$ better. 5 and 6 server configurations performed $78 \%$ and $88 \%$ better, but cost $22 \%$ and $38 \%$ more (respectively).

\section{References}

1. Smit, M., Nisbet, A., Stroulia, E., Edgar, A., Iszlai, G., Litoiu, M.: Capacity planning for service-oriented architectures. In: CASCON 2008: Proceedings of the 2008 conference of the Center for Advanced Studies on collaborative research, pp. 144156. ACM, New York (2008)

2. Smit, M., Nisbet, A., Stroulia, E., Iszlai, G., Edgar, A.: Toward a simulationgenerated knowledge base of service performance. In: Proceedings of the 4th International Workshop on Middleware for Service Oriented Computing (November 2009) 